4 Teresa Danielle Bergazin (ORCID: 0000-0002-0573-6178) ${ }^{1}$, Ido Y. Ben-Shalom (ORCID: 0000-0002-7237-1690 )², 5 Nathan M. Lim (ORCID: 0000-0002-2075-5852) ${ }^{1}$, Sam C. Gill (ORCID: 0000-0002-3743-3657) ${ }^{3}$, Michael K. Gilson 6 (ORCID: 0000-0002-3375-1738) ${ }^{2}$, David L. Mobley (ORCID: 0000-0002-1083-5533) ${ }^{1,3}$

$7{ }^{1}$ Department of Pharmaceutical Sciences, University of California, Irvine, Irvine, California 92697, United States;

$\therefore \quad 2$ Skaggs School of Pharmacy and Pharmaceutical Sciences, University of California, San Diego, La Jolla, California

- 92093, United States; ${ }^{3}$ Department of Chemistry, University of California, Irvine, Irvine, California 92697, United

10 States

11 *For correspondence:

12 dmobley@mobleylab.org (DM)

Abstract Water molecules can be found interacting with the surface and within cavities in proteins. However, water exchange between bulk and buried hydration sites can be slow compared to simulation timescales, thus leading to the inefficient sampling of the locations of water. This can pose problems for free energy calculations for computer-aided drug design. Here, we apply a hybrid method that combines nonequilibrium candidate Monte Carlo (NCMC) simulations and molecular dynamics (MD) to enhance sampling of water in specific areas of a system, such as the binding site of a protein. Our approach uses NCMC to gradually remove interactions between a selected water molecule and its environment, then translates the water to a new region, before turning the interactions back on. This approach of gradual removal of interactions, followed by a move and then reintroduction of interactions, allows the environment relax in response to the proposed water translation, improving acceptance of moves and thereby accelerating water exchange and sampling. We validate this approach on several test systems including the ligand-bound MUP-1 and HSP90 proteins with buried crystallographic waters removed. We show that our NCMC/MD method enhances water sampling relative to normal MD when applied to these systems. Thus, this approach provides a strategy to improve water sampling in molecular simulations which may be useful in practical applications in drug discovery and biomolecular design.

\title{
0.1 Keywords
}

Molecular Dynamics simulations · Monte Carlo · NCMC · nonequilibrium candidate Monte Carlo · enhanced sampling · water sampling · buried binding sites · buried cavity $\cdot$ buried water $\cdot$ Major Urinary Protein · Heat Shock Protein 90

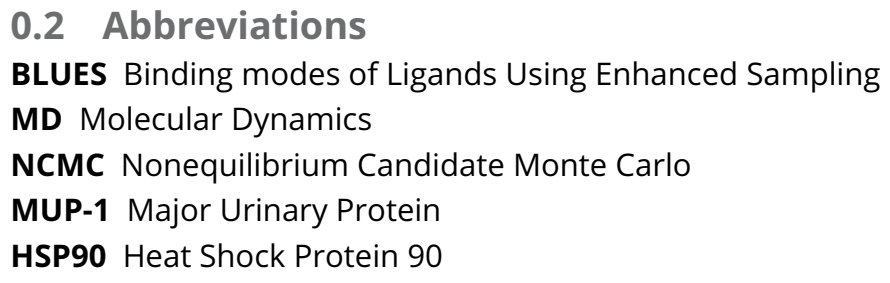




\section{Introduction}

Proteins are found in aqueous environments where water plays a major role in determining their structure, function, and dynamics [6, 24]. Water molecules can also be found in cavities in proteins $[18,28,36]$ where they play a variety of roles, such as facilitating receptor-ligand recognition and contributing to the stability of proteins $[7,9,24,36,43]$.

Classical molecular dynamics (MD) simulations can be used to understand the motions and interactions of biomolecular systems, including how proteins interact with water. However, water exchange between bulk and buried hydration sites can be slow compared to simulation timescales [13,27,31]. This leads to the inefficient sampling of the locations of water and water's role in binding events [15]. Simulations that do not account for these water motions will give an incomplete picture of the binding process and any downstream predictions will thus risk being in error [15, 31].

Several methods may better sample water occupancy and rearrangements in the cavities of proteins. Monte Carlo (MC) methods can substantially accelerate water sampling via large translational water moves around a system, but these MC moves can be difficult to get accepted due to steric clashes in the system. For example, grand canonical Monte Carlo [3, 4], which works by insertion and deletion of water to maintain a specific chemical potential, has been applied to sample water configurations and accelerate occupancy of buried sites [23, 38, 45]. However, this approach has been shown to be inefficient due to steric clashes which results in a high rejection of the proposed moves [29, 39]. Another approach integrates Metropolis MC translational water moves with traditional MD to equilibrate water across steric barriers and into buried hydration sites that are not accessible with pure MD [10].

Here, we seek to enhance the sampling of water rearrangements through extension of our Binding Modes of Ligands Using Enhanced Sampling (BLUES) approach [19], which combines hybrid nonequilibrium candidate Monte Carlo (NCMC) [34] with MD simulations. BLUES has been shown to enhance ligand sampling efficiency by more than two orders of magnitude compared to classical MD when applied to a model test system [19]. In BLUES, NCMC alchemically scales off the electrostatic and steric interactions until a water molecule is no longer interacting with its environment and then translates it to a new location before scaling the interactions back on. This results in a proposed NCMC move which is either accepted or rejected based on the integrated work during this process. After this, the NCMC move is followed by traditional molecular dynamics. By mixing NCMC translational water sampling moves with classical MD simulations, we improve water sampling in a selected region, such as a binding site of a protein, where water motions are known to be challenging or slow to sample and likely to pose problems for calculations of interest, such as free energy calculations [15]. In this work, we use the BLUES framework to exchange waters around a specified region of a system. Here, we focus on testing it in specific contexts where water rearrangements can pose challenges for MD sampling, such as buried binding sites in proteins.

\section{Methods}

We introduce a method that integrates NCMC translational water moves with classical MD, allowing water molecules to hydrate buried sites. Here, we detail how this approach is implemented and tested.

\subsection{Implementation of NCMC/MD in BLUES}

BLUES (Binding Modes of Ligands Using Enhanced Sampling), which combines NCMC with classical MD, was originally created to enhance the sampling of ligand binding modes [19], but has begun applying the same techniques to enhance sampling of other degrees of freedom also important in ligand binding, such as sidechain rearrangements [11] and, here, water motions. In BLUES, NCMC moves are executed through a switching protocol that is comprised of a series of perturbation and propagation/relaxation steps involving structural and dynamic degrees of freedom [34]. This process helps lower possible steric or electrostatic clashes by allowing the environment surrounding the perturbed region to relax around the proposed state.

NCMC moves are implemented by alchemically "turning off" the interactions between an object in the system and its surrounding environment before the move, as detailed in Figure 1. First, the electrostatic and then the steric interactions are turned off and on by scaling $\lambda$, a variable that controls the strength of nonbonded interactions, from 1 (fully interacting state) to 0 (noninteracting state) over a user-determined number of $n$ NCMC steps. At the point where the object is noninteracting, the target object's atoms are repositioned (while the internal coordinates/conformation remain the same during this move) and then the interactions are scaled back on until $\lambda=1$ in reverse order (first sterics and then electrostatics). The total work done during this process is summed and used to either accept or reject the proposed move (following a modified Metropolis-Hastings acceptance criterion [28] to maintain detailed balance). The NCMC move is then followed by a user-determined number of MD steps. Additional details of BLUES are described in the work of Gill et al. [19]. 
A

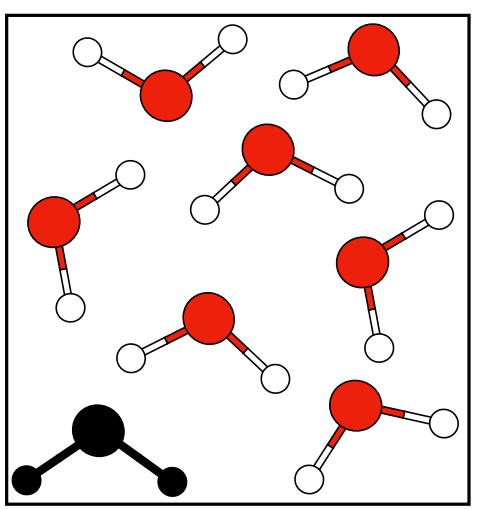

D

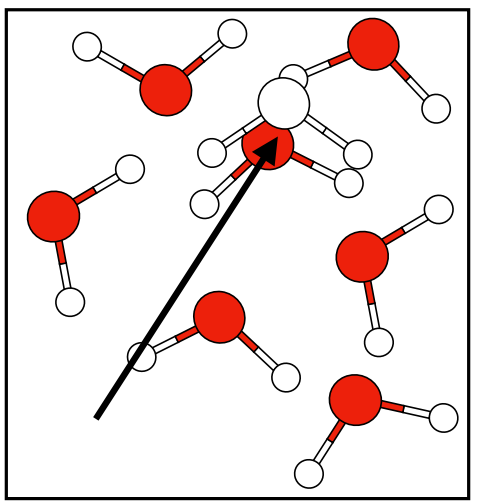

B

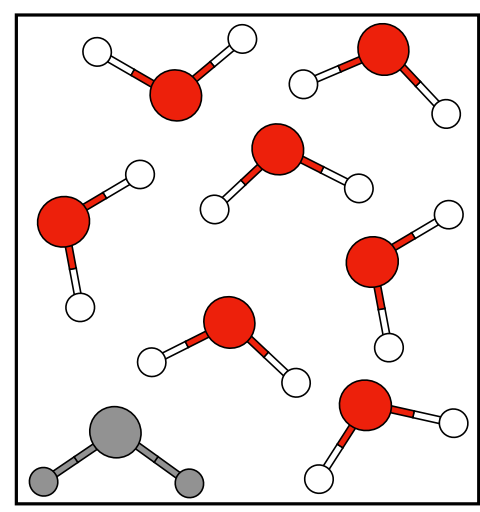

$\mathbf{E}$

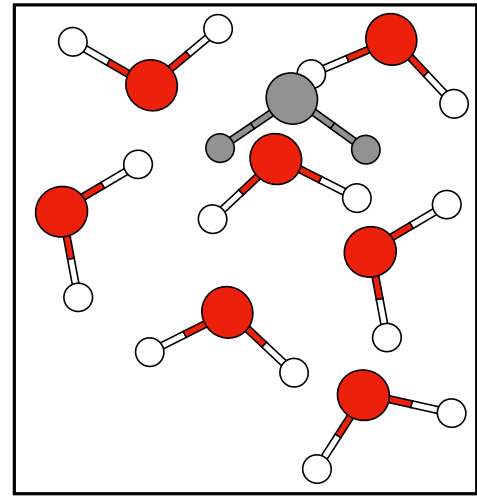

C

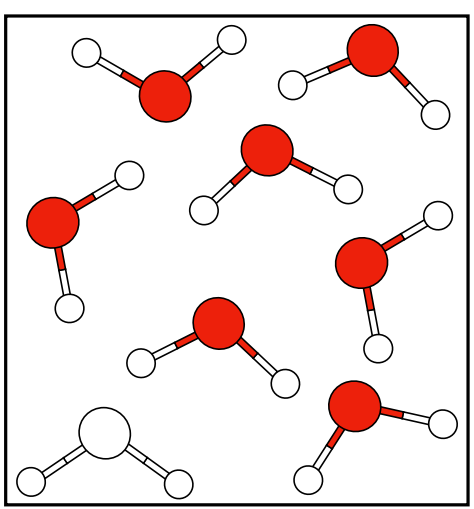

$\mathbf{F}$

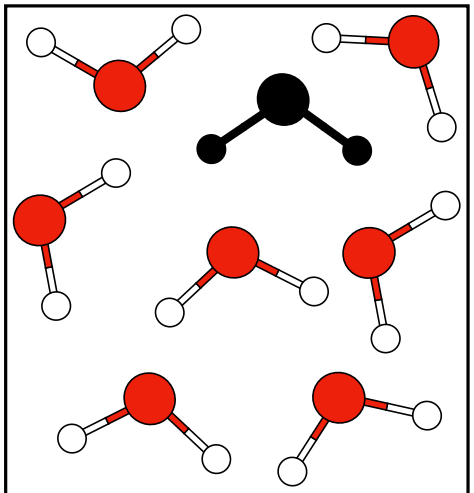

Figure 1. Molecular interactions between atoms are turned off and on during an NCMC translational water move. In this cartoon, water molecules are represented here by red and white spheres for the oxygen and hydrogen atoms. The black-filled water represents a fully interacting water molecule that has been selected to be moved. Gray-filled water represents intermediate levels of interaction and white-filled represents the fully non-interacting water molecule. A) The water molecule (in black) is fully interacting with its surrounding environment, and in this case, other water molecules. B) The water's interactions are partially off, allowing the other water molecules to slightly relax. C) The water's interactions are fully turned off. D) The water is randomly translated to somewhere else in the system (indicated by a black arrow) with its interactions remaining off. E) The water's interactions are partially turned on and the propagation steps of NCMC allow relaxation of the translated water and its surroundings to resolve clashes. F) At the end of the NCMC protocol, the water molecule is once again in the fully interacting state and in a new location. This entire process comprises a proposed move, which is accepted or rejected based on the nonequilibrium work done in this process, and then followed by conventional MD. 


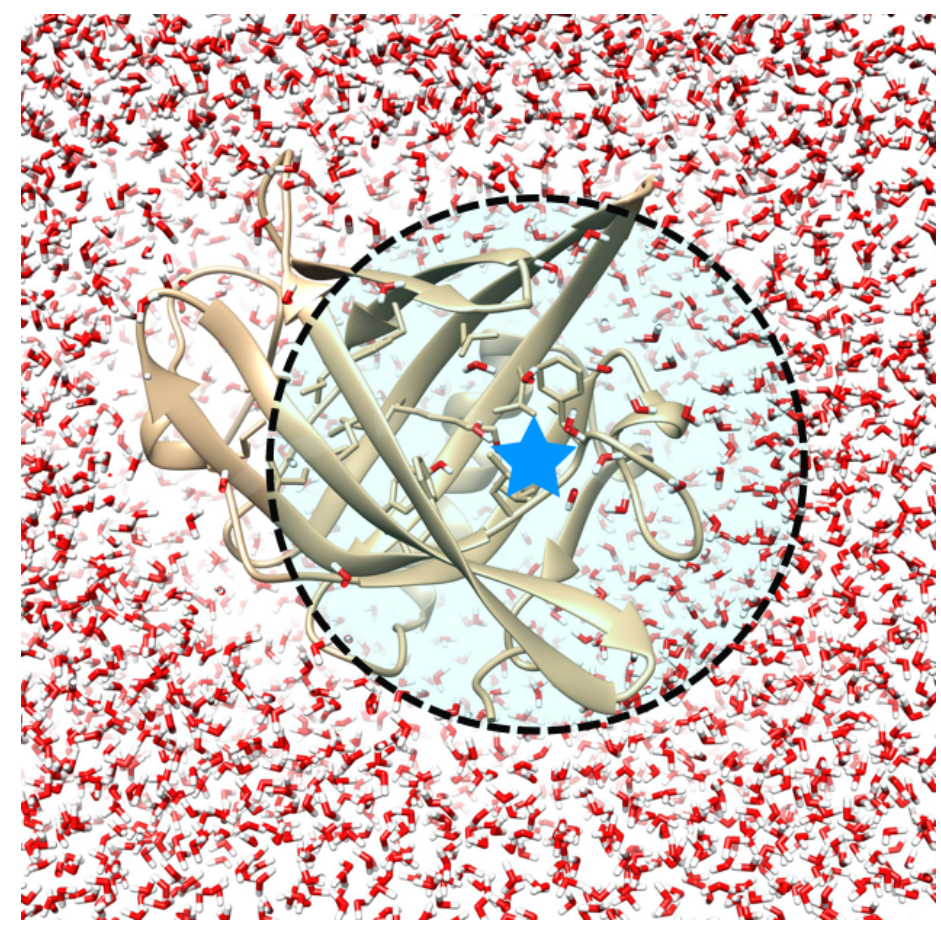

Figure 2. Example of a user-defined radius that covers a particular area of interest. Here, the MUP-1 protein-ligand system is shown. The radius used (indicated by the black dashed line) defines a sphere around a user-selected atom (represented by a blue star) in the system, such as an atom inside the binding site of a protein.

\subsection{Translational water moves with BLUES}

Here, we build upon the BLUES framework by incorporating "water hopping" moves where random water molecules can be translated between bulk and within a region via NCMC move proposals. Water hopping moves were created in order to enhance sampling of key hydration sites such as in water bridging locations between a protein and ligand, particularly in buried cavities inaccessible from bulk water.

To define a region within which the water hops occur, the user selects an atom as the center and defines a radius to generate a sphere which encompasses the area of interest (Figure 2). This area of interest must be large enough to include some bulk water to allow water exchange. Our algorithm will subsequently use this radius to select a random water molecule and propose moving it to a random new position within this region. Specifically, for each NCMC move proposal, a random water molecule is selected, a random point in this region is generated, the interactions are scaled off between the atoms of the water molecule and its surrounding environment, then the water molecule is translated to the new location defined by the random point, and then the interactions are scaled back on (Figure 3). The work done during this NCMC move proposal is accumulated and the move is either accepted or rejected using the Metropolis-Hastings acceptance criterion [28]. Further water hopping implementation details are available in python scripts deposited in the Supporting Information.

\subsection{Comparing sampling efficiency using the number of force evaluations}

BLUES simulations consist of intervals of both classical MD and NCMC, so comparing BLUES to classical MD requires accounting for the cost of the switching protocol that occurs during the NCMC move. We account for the additional cost from NCMC by considering the number of force evaluations rather than the aggregated simulation time in nanoseconds or microseconds.

NCMC carries out a single force evaluation for each perturbation or propagation/relaxation step. The perturbation steps are the instantaneous perturbation of the water molecules coordinates (or for turning off/on the alchemical parameters), and this is combined with propagation steps via Langevin dynamics [19]. In other words, perturbation steps modify the system or its potential, and propagation steps propagate the dynamics. A BLUES simulation consists of NCMC and MD, so a BLUES simulation will have a total cost in force evaluations of: 


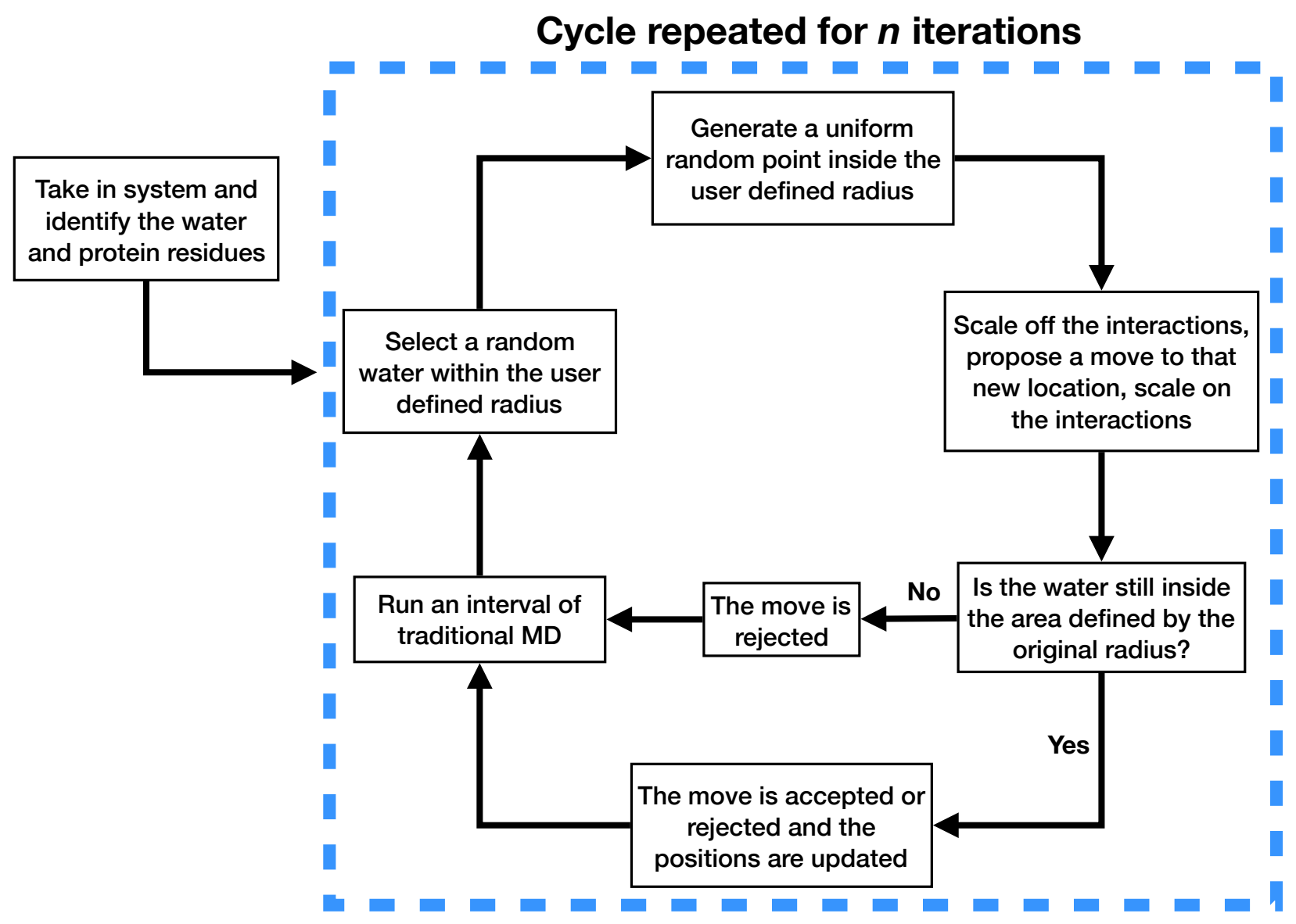

Figure 3. Workflow of BLUES water hopping proposals. Before any water is translated to a new location, the user first selects an atom and picks a radius defining a sphere encompassing an area of interest around the position of the atom and BLUES identifies all the water and protein residues in the system. Afterward, BLUES goes through a number of cycles for $n$ iterations, where each cycle is as shown inside the dashed box. 
A

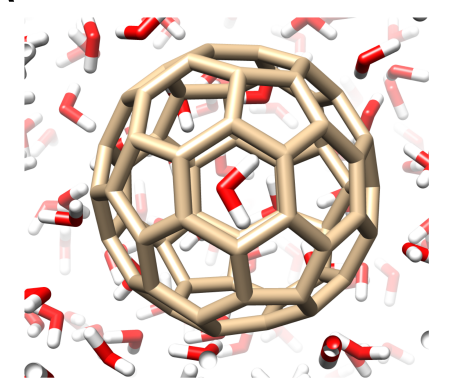

B

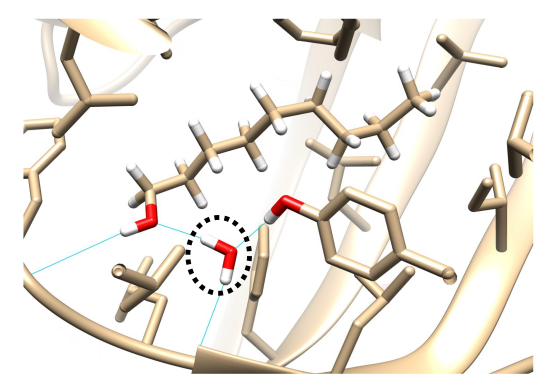

C

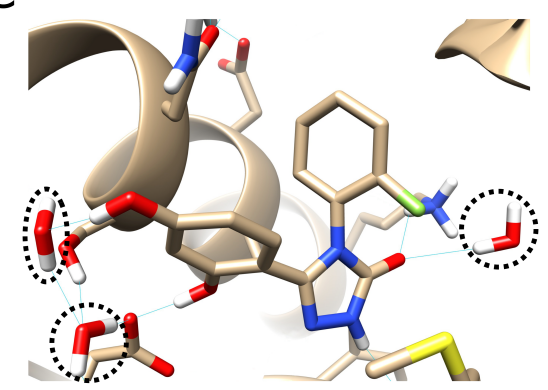

Figure 4. Systems used to test the ability of NCMC/MD water hopping to allow the exchange of water. (A) A $C_{60}$ buckyball with a single trapped water molecule. (B) The buried hydration site of the MUP-1 protein with a bound ligand. (C) The hydration site of the HSP90 protein bound to a ligand. The protein-ligand systems have internal water(s) (indicated by the black dashed line) that do not easily exchange with bulk.

where $n$ Steps MD is the number of MD steps per iteration, nSteps NCMC is the number of NCMC steps per iteration and n/ter is the number cycles consisting of NCMC move proposals followed by MD. The total cost in force evaluations for classical MD is equivalent to the total number of MD steps.

\subsection{Test cases and simulation details}

We used a $\mathrm{C}_{60}$ buckyball, a water box system with dividing graphene sheets, Major Urinary Protein (MUP-1) and Heat Shock Protein 90 (HSP90) as systems to test the ability of the NCMC/MD water hopping moves to enhance the sampling of water molecules in desired regions. Many of these systems were also used in a similar study to validate Metropolis MC translational water moves with traditional MD [10].

The first system was a $\mathrm{C}_{60}$ buckyball with a water molecule trapped inside (Figure 4.A). This water molecule is unable to interact with bulk water and cannot form any hydrogen bonds with the buckyball's carbon atoms. Hence, it is in an energetically unfavorable environment, but it is unable to diffuse out. We chose a sampling region that was centered on a carbon atom in the buckyball and extended $12 \AA$ out, such that the region included the entire buckyball and some bulk water. The box size was $\sim 44 \times 44 \times 44 \AA^{3}$, and had a total of 213 water molecules.

The second system was a rectangular water box divided into two regions by impermeable planar graphene sheets (Figure 5.A). These two regions had initially different water densities where the outer and inner region had densities of about 21.5 water $/ \mathrm{nm}^{3}$ and 18.5 water $/ \mathrm{nm}^{3}$, respectively. The rectangular box was $\sim 32 \times 32 \times 85 \AA^{3}$ and the system had a total of 1915 water molecules. The initially differing densities between the outer and inner region tested the ability of the NCMC/MD water hopping method to equalize the water densities between the sheets. We chose a sampling region that was centered on a carbon atom in the middle of one of the sheets and extended $15 \AA$ out so that the sampling region covered the same amount of area in the inner and outer regions. This choice was important to ensure that we didn't make dramatically more move proposals to one region relative to the other. Additionally, we chose our sampling region so that it did not extend outside of the simulation box, thus avoiding issues where we might place waters in the same region more than once due to periodic boundary conditions, leading to artifacts.

The third and fourth systems tested the method's ability to exchange water between bulk and buried sites in two proteins. The third system was the MUP-1 protein [42] which contains a buried crystallographic water molecule that bridges between the ligand and the protein (Figure 4.B). The crystallographic water molecule was removed in order to test the ability of our water hopping moves to hydrate the buried cavity and reform the water bridging interaction. We chose a sampling region that was centered on a carbon atom in the ligand and extended $20 \AA$ out to include some bulk water (Figure 2). The box was $\sim 70 \times 70 \mathrm{x}$ $70 \AA^{3}$ the system had a total of 8,678 water molecules. The fourth system was the HSP90 protein (PDBID:5J64) [5] bound to a ligand which forms interactions with the protein through three bridging water molecules, as shown in Figure 4.C. The box was $\sim 82 \times 82 \times 82 \AA^{3}$ and the system had a total of 13,831 water molecules. We chose a sampling region that was centered on a carbon atom in the ligand, and extended $15 \AA$ out to include some bulk water.

The simulation boxes were built using tleap from AmberTools [12]. All of the systems used, where appropriate, the protein and ligand force field parameters from AMBER ff14SB [21, 26] and GAFF [44], respectively. The water molecules were parameterized using the TIP3P water model [22] in all cases. MD and BLUES simulations were performed using OpenMM (version 
A

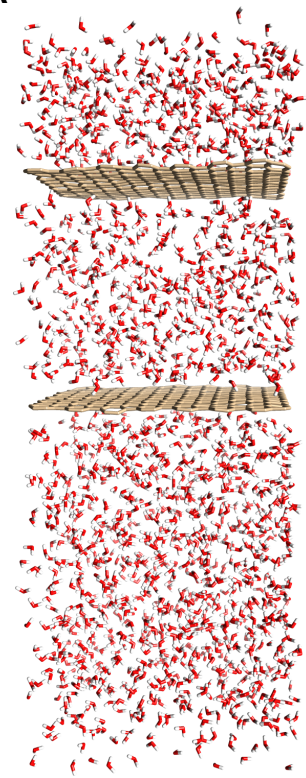

B

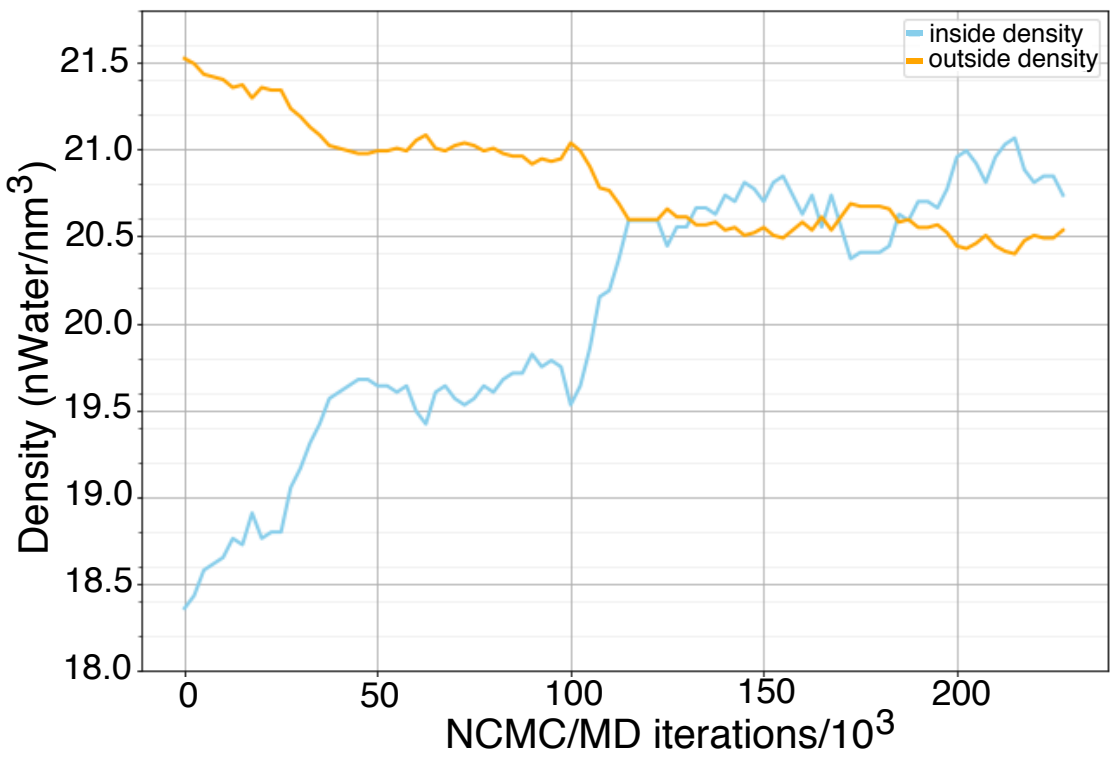

7.1.1) $[16,17]$. The systems were minimized until forces were below a tolerance of $10 \mathrm{~kJ} / \mathrm{mol}$. Long-range electrostatics were calculated using Particle Mesh Ewald [14]. Simulations were run using the hydrogen mass repartitioning scheme with 4 femtosecond timesteps [20].

To focus on water exchange the $\alpha$-Carbons and ligands in the protein ligand systems were restrained with a force constant of $5 \mathrm{kcal} / \mathrm{mol} \cdot \AA^{2}$, thus keeping the protein cavities from quickly collapsing. The carbon atoms in the buckyball and graphene walls in the water box system were also restrained with the same force constant as the protein-ligand systems, which held the buckyball in place and kept the graphene walls from collapsing/folding.

The temperature was set to $300 \mathrm{~K}$ in all cases except the water box with graphene sheets, which was set to $500 \mathrm{~K}$ so that the water in the system was less dense than liquid water and wouldn't form water droplets; thus, increasing the NCMC/MD water move acceptance rate so that any possible errors due to the method would be obvious. For the Buckyball system, equilibration consisted of 250 ps of NVT MD and 10 ns of NPT MD of equilibration. For the water box with dividing graphene sheets, equilibration consisted of 5 ns NVT MD. The MUP-1 system was equilibrated for 1 ns of NVT MD and 10ns NPT MD. The MD production run for the water box with dividing graphene sheets and the MUP-1 system was for 40 ns in the NPT ensemble. The HSP90 system was equilibrated for 1 ns of NVT MD and 80 ns NPT MD. The MD production run for HSP90 was for 285 ns in the NPT ensemble.

A BLUES simulation consists of a number of BLUES iterations, where each BLUES iteration is composed of a certain number of NCMC perturbation and propogation/relaxation steps (wherein the electrostatic and steric interactions are alchemically scaled off/on) followed by traditional MD. Here, we used the same amount of NCMC steps for all of the systems (except MUP-1, detailed below). For the water box system with dividing graphene sheets, BLUES with translational water moves was executed for 240,000 iterations, with each iteration consisting of 2,500 NCMC steps and 1,000 MD steps. The buckyball system was simulated for a total of 1,000 iterations, using 2,500 NCMC and 1,000 MD steps per iteration. Both of the solvated MUP-1 and HSP90 systems were simulated for a total of 10,000 BLUES iterations. For the MUP-1 system, 1,250, 2,500, 5,000 and 30,000 NCMC steps per iteration were tested to see how the number of NCMC steps affects the rate of water transfer from from bulk to the internal hydration site. The number of MD steps in all cases was 1,000 MD steps per iteration. For the HSP90 system, each BLUES iteration consisted of 2,500 NCMC steps and 1,000 MD steps. Further simulation details are available in scripts deposited in the SI. 


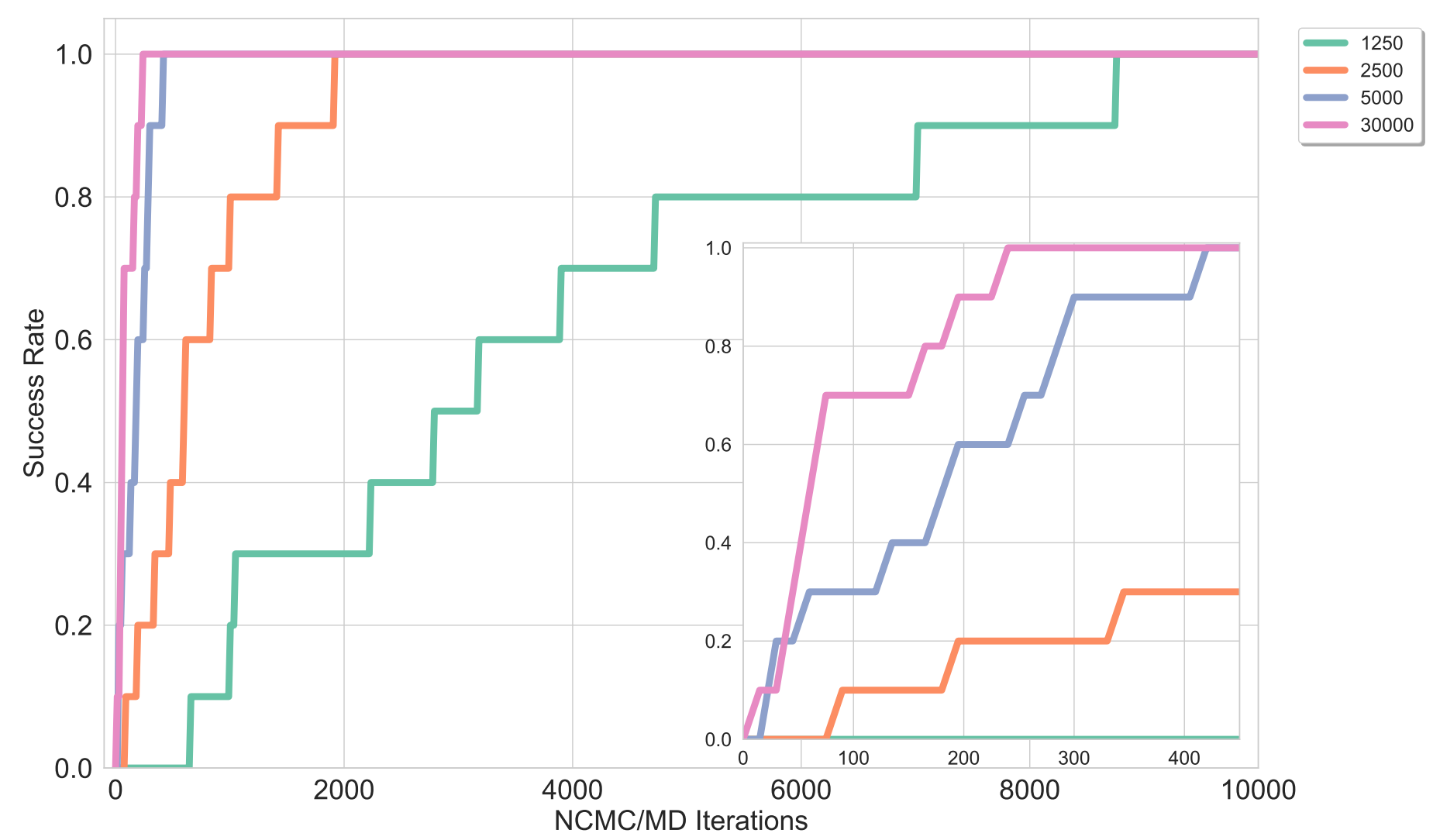

Figure 6. Increasing the amount of NCMC steps increases the rate of water transfer from bulk to the internal hydration site in MUP1. Ten replicate simulations with different random seed numbers were run for each NCMC value. All of the simulations were run for 10,000 iterations, with each iteration consisting of a certain number of steps of NCMC and MD. The different colors indicate various amounts of NCMC steps used. The success rate is equivalent to the ratio between the sites that have been hydrated and the total number. (A) shows that using a lower NCMC amount increases the number of BLUES iterations (or cycles) for the cavity to become hydrated, such as 1250 (green) and 2500 (orange). The inset, (B), zooms in on the success rate at low iteration number and shows that increasing the amount of NCMC steps decreases the number of iterations needed. 5000 (blue) NCMC steps needed a little more than 400 BLUES iterations to hydrate the cavity and 30000 (pink) NCMC steps needed no more than 250 BLUES iterations to hydrate the cavity. 


\section{Results and Discussion}

The hybrid BLUES (NCMC/MD) approach described here accelerates water sampling during simulations by incorporating translational water moves during the NCMC component of each NCMC/MD cycle. We refer to these translational water moves in BLUES as "water hopping". Here, we tested these water hopping moves in a range of systems. Particularly, we use a $\mathrm{C}_{60}$ buckyball, water box system with dividing graphene sheets, MUP-1 and HSP90 protein-ligand systems to validate the water hopping methodology. Across all of the systems tested, we find that BLUES (NCMC/MD) water hopping moves allowed water exchange between regions, while plain MD did not.

The first test system was a $\mathrm{C}_{60}$ buckyball simulated in bulk water, with a single water molecule housed inside (Figure 4.A). Inside of the buckyball, the water molecule is in an energetically unstable environment relative to a water molecule in bulk. We find that water hopping moves can relocate the water molecule from the inside of the buckyball to bulk water, as expected. Since the trapped water molecule is unable to interact with bulk water or form hydrogen bonds with the buckyball's carbon shell, it is thermodynamically favorable for it to escape, but it is unable to do so with conventional MD. We chose a sampling region centered on a carbon atom in the buckyball so that the sampling region encompassed the buckyball and some bulk water. While the water molecule is not able to escape the buckyball with plain MD [10], water hopping allowed the water molecule to escape, returning it to the surrounding bulk water after $2.1 \times 10^{5}$ force evaluations. The buckyball remains unoccupied after the water molecule leaves.

The second test system was a water box system divided into two regions by impermeable graphene sheets (Figure 5.A), with each region having different initial water densities. We find that water hopping successfully equalizes the water between the two regions (Figure 5.B). We chose a sampling region centered on a carbon atom in the middle of one of the graphene sheets, such that the sampling region encompassed equivalent amounts of both the inner and outer regions. The relative densities of each region initially differed, but should become uniform over time if BLUES is allowing waters to hop between the two regions. Standard MD does not allow water to enter the inner region between the graphene sheets because the sheets act as barriers that prevent water from passing through them. However, we find that translational water moves in BLUES allow water molecules to hop across the sheets, causing the densities to gradually equalize in both regions (Figure 5.B). Here we found this took $4.2 \times 10^{8}$ force evaluations.

Next, we examined a buried hydration site in MUP-1, which has a buried crystallographic water molecule that bridges between the ligand and the protein (Figure 4.B). The crystallographic water molecule was removed from the buried site and water hopping successfully rehydrated it. We chose a sampling region that was centered on an atom in the ligand and extended out to include some bulk water (such as in Figure 2), such that the sampling region encompassed the buried hydration site and had access to bulk. With plain MD the water did not resume its crystallographic bridging position even after $1.5 \mu \mathrm{s}$, equivalent to $3.8 \times 10^{8}$ force evaluations. However, BLUES was able to recover the crystallographic water. On average (across 11 replicates), it took BLUES $2.6 \times 10^{6}$ force evaluations to hydrate the site (using 2,500 NCMC steps and 1,000 MD steps per iteration), and no water moves were accepted that dehydrated the site. Additionally, we tested how the number of NCMC steps per iteration affects the rate of water transfer to the hydration site by simulating with 1,250,2,500,5,000, and 30,000 NCMC steps per iteration, and used 1,000 MD steps per iteration for each. As expected, increasing the number of NCMC steps per iteration increases the rate of water transfer from bulk to the buried hydration site in MUP-1, as seen in Figure 6.

Although increasing the number of NCMC steps per iteration decreases the number of NCMC/MD iterations required for the site to become hydrated, we find that increasing the number of NCMC steps per iteration also negatively effects the efficiency of the water hopping in hydrating the cavity (Table S2). Eventually, the increase in efficiency from allowing more relaxation is swamped by the associated increase in computational cost. However, relatively small amounts of relaxation have considerable payoff, resulting in a sort of sweet spot in terms of amount of relaxation. We find that here, roughly 1250 NCMC steps per iteration is a good compromise to ensure water hopping is as efficient as possible.

Lastly, we examined three hydration sites in the binding site region of the HSP90 protein-ligand system (Figure 4.C). All three crystallographic water molecules were removed from the hydration site in the HSP90 system and water hopping successfully rehydrated each hydration site. We chose a sampling region that was centered on a ligand atom and extended out to encompass the buried hydration site, ligand and some bulk water. With plain MD, only one out of the three water molecules were able to resume the crystallographic bridging positions within $285 \mathrm{~ns}$, which is equivalent to $7.1 \times 10^{7}$ force evaluations. This water molecule moved in from a starting position in bulk water. It took BLUES 5.9 $10^{6}$ force evaluations on average (across 4 replicates) to occupy all three of the hydration sites. After the buried cavity had been hydrated, no NCMC moves were accepted that removed any of the water molecules, indicating that the occupancy of these sites is favorable. 
The sampling region used for the protein-ligand systems encapsulates the binding pocket and some bulk water. Relative to $M D$, we find that we can increase efficiency by making the area of interest the focal point of NCMC move attempts. Making the sampling region just large enough to cover a specific ligand-binding site and bulk water allows us to speed up the equilibration of water between these two regions, and this strategy has been successfully used elsewhere [10] If the sampling region covered a greater amount of bulk water in these cases, the efficiency would decrease because the equilibration of water between regions would be slower as more water moves would move water molecules around in just bulk water. In general, we recommend setting the radius to be as small as possible while ensuring that the particular area of interest and some bulk is covered, thus increasing efficiency. In some cases, a larger sampling region may be more desirable, such as a protein with multiple hydration cavities, and this would simply require defining a larger sampling region which covers all of the cavities. Additionally, the user must be careful when defining the sampling region when using periodic boundary conditions. If the radius is set to encompass any area outside of the box, and periodic boundary conditions are used, there could be overlapping regions in the sampling area and this will result in more water moves being proposed to those areas, creating problems as noted above.

Water hopping could be used to discover important hydration sites in proteins. Crystallography does not always provide an accurate view of water positions and occupancies [35]. Only relatively highly ordered waters can be resolved in crystal structures, which may be a small subset of all waters which are present. Additionally, partial and weak density can obscure determination of where water molecules are present. At the same time, waters can be critical in protein dynamics $[41,47]$ and for the thermodynamics of ligand binding $s[1,2,8,25,30,32,33,37,46]$, meaning that treatment of such waters - even when not obvious from experimental data - can be critical. Our method could explore such feasible hydration sites as well as the orientation of critical water molecules in cases where structural data is ambiguous.

\section{Conclusions and Future Work}

In this study, we implemented water hopping moves within our BLUES (NCMC+MD) framework to enhance the sampling of water rearrangements relative to traditional MD for systems that have buried hydration sites.

We validated BLUES with translational water moves on a water box with dividing graphene sheets, a buckyball with an energetically unfavorable water trapped inside, and both the MUP-1 and HSP90 proteins bound to a ligand with crystallographic bridging water removed. We then evaluate the efficiency of BLUES in hydrating the sites in the protein-ligand systems, based on the number of force evaluations. Overall, we demonstrate that NCMC enhances sampling relative to normal MD.

This water hopping approach can be used to find areas that are likely to be populated by waters in protein binding sites and sample water rearrangements potentially more efficiently than traditional MD. Water hopping moves could be combined with additional types of BLUES moves such as ligand [19,40] or sidechain [11] rotational moves for broader applications.

The size of the sampling region is an important parameter in our method, and one we intend to optimize in the future. In the future, additional work could be done to help improve the acceptance of water hopping moves. To improve the acceptance and increase the efficiency of NCMC/MD translational water moves, move proposals could be made to be more selective. In the current work, the move proposals can be made anywhere that is encompassed by the radius. To make the move more efficient, water hopping could be redesigned to help reduce move proposals that only move water molecules around in bulk, thus focusing on move proposals to the interior of the protein using methods like those detailed in the work of Ben-Shalom et al. [10]. Other work could include slight changes to how the sampling region gets defined. Rather than having the user have to manually define a specific atom, the sampling region could be made to automatically span from the center of mass of a protein or ligand. Additional work could also include comparisons of NCMC/MD water hopping to MC/MD water hopping, allowing us to test whether or not NCMC enhances sampling relative to MC; here, we compared only with traditional MD.

Overall, here, we introduced and validated our new water hopping approach to enhanced sampling of water rearrangements in BLUES, and find it is more efficient than standard MD on a by-force-evaluation basis for the systems considered here.

\section{Code and Data Availability}

The Supporting Information is available free of charge on https://github.com/MobleyLab/blues-water-hopping-paper and includes the code, scripts and input files used in this work.

\section{Acknowledgments}

TDB acknowledges support from the ACM SIGHPC/Intel Fellowship. DLM appreciates financial support from the National Institutes of Health (1R01GM108889-01) and the National Science Foundation (CHE 1352608). MKG acknowledges funding from the 
National Institute of General Medical Sciences (GM61300). The contents of this paper are solely the responsibility of the authors and do not necessarily represent the official views of the $\mathrm{NIH}$.

\section{Disclaimers}

The content is solely the responsibility of the authors and does not necessarily represent the official views of the National Institutes of Health.

\section{Disclosures}

DLM is a member of the Scientific Advisory Board of OpenEye Scientific Software and an Open Science Fellow with Silicon Therapeutics. MKG has an equity interest in and is a cofounder and scientific advisor of VeraChem LLC.

\section{References}

[1] Abel, R., Salam, N. K., Shelley, J., Farid, R., Friesner, R. A., and Sherman, W. (2011). Contribution of Explicit Solvent Effects to the Binding Affinity of Small-Molecule Inhibitors in Blood Coagulation Factor Serine Proteases. ChemMedChem, 6(6):1049-1066.

[2] Abel, R., Young, T., Farid, R., Berne, B. J., and Friesner, R. A. (2008). Role of the Active-Site Solvent in the Thermodynamics of Factor Xa Ligand Binding. J. Am. Chem. Soc., 130(9):2817-2831.

[3] Adams, D. (1974). Chemical potential of hard-sphere fluids by Monte Carlo methods. Molecular Physics, 28(5):1241-1252.

[4] Adams, D. (1975). Grand canonical ensemble Monte Carlo for a Lennard-Jones fluid. Molecular Physics, 29(1):307-311.

[5] Amaral, M., Kokh, D. B., Bomke, J., Wegener, A., Buchstaller, H. P., Eggenweiler, H. M., Matias, P., Sirrenberg, C., Wade, R. C., and Frech, M. (2017). Protein conformational flexibility modulates kinetics and thermodynamics of drug binding. Nat Commun, 8(1):2276.

[6] Ball, P. (2008). Water as an Active Constituent in Cell Biology. Chem. Rev., 108(1):74-108.

[7] Baron, R., Setny, P., and McCammon, J. A. (2010). Water in Cavity-Ligand Recognition. J. Am. Chem. Soc., 132(34):12091-12097.

[8] Bayden, A. S., Moustakas, D. T., Joseph-McCarthy, D., and Lamb, M. L. (2015). Evaluating Free Energies of Binding and Conservation of Crystallographic Waters Using SZMAP. J. Chem. Inf. Model., 55(8):1552-1565.

[9] Bellissent-Funel, M.-C., Hassanali, A., Havenith, M., Henchman, R., Pohl, P., Sterpone, F., van der Spoel, D., Xu, Y., and Garcia, A. E. (2016). Water Determines the Structure and Dynamics of Proteins. Chem. Rev., 116(13):7673-7697.

[10] Ben-Shalom, I. Y., Lin, C., Kurtzman, T., Walker, R. C., and Gilson, M. K. (2019). Simulating Water Exchange to Buried Binding Sites. J. Chem. Theory Comput., 15(4):2684-2691.

[11] Burley, K. H., Gill, S. C., Lim, N. M., and Mobley, D. L. (2019). Enhancing Side Chain Rotamer Sampling Using Nonequilibrium Candidate Monte Carlo. J. Chem. Theory Comput., 15(3):1848-1862.

[12] Case, D. A., Cheatham, T. E., Darden, T., Gohlke, H., Luo, R., Merz, K. M., Onufriev, A., Simmerling, C., Wang, B., and Woods, R. J. (2005). The Amber biomolecular simulation programs. J. Comput. Chem., 26(16):1668-1688.

[13] Cournia, Z., Allen, B., and Sherman, W. (2017). Relative Binding Free Energy Calculations in Drug Discovery: Recent Advances and Practical Considerations. J. Chem. Inf. Model., 57(12):2911-2937.

[14] Darden, T., York, D., and Pedersen, L. (1993). Particle mesh Ewald: An $N \cdot \log (N)$ method for Ewald sums in large systems. The Journal of Chemical Physics, 98(12):10089-10092.

[15] Deng, Y. and Roux, B. (2008). Computation of binding free energy with molecular dynamics and grand canonical Monte Carlo simulations. The Journal of Chemical Physics, 128(11):115103.

[16] Eastman, P., Friedrichs, M. S., Chodera, J. D., Radmer, R. J., Bruns, C. M., Ku, J. P., Beauchamp, K. A., Lane, T. J., Wang, L.-P., Shukla, D., Tye, T., Houston, M., Stich, T., Klein, C., Shirts, M. R., and Pande, V. S. (2013). OpenMM 4: A Reusable, Extensible, Hardware Independent Library for High Performance Molecular Simulation. J. Chem. Theory Comput., 9(1):461-469.

[17] Eastman, P., Swails, J., Chodera, J. D., McGibbon, R. T., Zhao, Y., Beauchamp, K. A., Wang, L.-P., Simmonett, A. C., Harrigan, M. P., Stern, C. D., Wiewiora, R. P., Brooks, B. R., and Pande, V. S. (2017). OpenMM 7: Rapid development of high performance algorithms for molecular dynamics. PLOS Comput Biol, 13(7):e1005659.

[18] Ernst, J., Clubb, R., Zhou, H., Gronenborn, A., and Clore, G. (1995). Demonstration of positionally disordered water within a protein hydrophobic cavity by NMR. Science, 267(5205):1813-1817. 
19] Gill, S. C., Lim, N. M., Grinaway, P. B., Rustenburg, A. S., Fass, J., Ross, G. A., Chodera, J. D., and Mobley, D. L. (2018). Binding Modes of Ligands Using Enhanced Sampling (BLUES): Rapid Decorrelation of Ligand Binding Modes via Nonequilibrium Candidate Monte Carlo. J. Phys. Chem. $B, 122(21): 5579-5598$.

[20] Hopkins, C. W., Le Grand, S., Walker, R. C., and Roitberg, A. E. (2015). Long-Time-Step Molecular Dynamics through Hydrogen Mass Repartitioning. J. Chem. Theory Comput., 11(4):1864-1874.

[21] Hornak, V., Abel, R., Okur, A., Strockbine, B., Roitberg, A., and Simmerling, C. (2006). Comparison of multiple Amber force fields and development of improved protein backbone parameters. Proteins, 65(3):712-725.

[22] Jorgensen, W. L., Chandrasekhar, J., Madura, J. D., Impey, R. W., and Klein, M. L. (1983). Comparison of simple potential functions for simulating liquid water. The Journal of Chemical Physics, 79(2):926-935.

[23] Lakkaraju, S. K., Raman, E. P., Yu, W., and MacKerell, A. D. (2014). Sampling of Organic Solutes in Aqueous and Heterogeneous Environments Using Oscillating Excess Chemical Potentials in Grand Canonical-like Monte Carlo-Molecular Dynamics Simulations. J. Chem. Theory Comput., 10(6):2281-2290.

[24] Levy, Y. and Onuchic, J. N. (2006). WATER MEDIATION IN PROTEIN FOLDING AND MOLECULAR RECOGNITION. Annu. Rev. Biophys. Biomol. Struct., 35(1):389-415.

[25] Li, Z. and Lazaridis, T. (2012). Computing the Thermodynamic Contributions of Interfacial Water. In Baron, R., editor, Computational Drug Discovery and Design, volume 819, pages 393-404. Springer New York, New York, NY. Series Title: Methods in Molecular Biology.

[26] Maier, J. A., Martinez, C., Kasavajhala, K., Wickstrom, L., Hauser, K. E., and Simmerling, C. (2015). ff14SB: Improving the Accuracy of Protein Side Chain and Backbone Parameters from ff99SB. J. Chem. Theory Comput., 11(8):3696-3713.

[27] Maurer, M., de Beer, S., and Oostenbrink, C. (2016). Calculation of Relative Binding Free Energy in the Water-Filled Active Site of OligopeptideBinding Protein A. Molecules, 21(4):499.

[28] Meyer, E. (1992). Internal water molecules and H-bonding in biological macromolecules: A review of structural features with functional implications. Protein Sci., 1(12):1543-1562.

[29] Mezei, M. (1980). A cavity-biased ( $T, V, \mu)$ Monte Carlo method for the computer simulation of fluids. Molecular Physics, 40(4):901-906.

[30] Michel, J., Tirado-Rives, J., and Jorgensen, W. L. (2009). Prediction of the Water Content in Protein Binding Sites. J. Phys. Chem. B, 113(40):13337-13346.

[31] Mobley, D. L. and Gilson, M. K. (2017). Predicting Binding Free Energies: Frontiers and Benchmarks. Annu. Rev. Biophys., 46(1):531-558.

[32] Nguyen, C. N., Cruz, A., Gilson, M. K., and Kurtzman, T. (2014). Thermodynamics of Water in an Enzyme Active Site: Grid-Based Hydration Analysis of Coagulation Factor Xa. J. Chem. Theory Comput., 10(7):2769-2780.

[33] Nguyen, C. N., Kurtzman Young, T., and Gilson, M. K. (2012). Grid inhomogeneous solvation theory: Hydration structure and thermodynamics of the miniature receptor cucurbit[7]uril. The Journal of Chemical Physics, 137(4):044101.

[34] Nilmeier, J. P., Crooks, G. E., Minh, D. D. L., and Chodera, J. D. (2011). Nonequilibrium candidate Monte Carlo is an efficient tool for equilibrium simulation. Proceedings of the National Academy of Sciences, 108(45):E1009-E1018.

[35] Nittinger, E., Schneider, N., Lange, G., and Rarey, M. (2015). Evidence of Water Molecules-A Statistical Evaluation of Water Molecules Based on Electron Density. J. Chem. Inf. Model., 55(4):771-783.

[36] Park, S. and Saven, J. G. (2005). Statistical and molecular dynamics studies of buried waters in globular proteins. Proteins, 60(3):450-463.

[37] Pearlstein, R. A., Sherman, W., and Abel, R. (2013). Contributions of water transfer energy to protein-ligand association and dissociation barriers: Watermap analysis of a series of p38 $\alpha$ MAP kinase inhibitors: Water Transfer in Structure-Kinetic Relationships. Proteins, 81(9):15091526.

[38] Ross, G. A., Bodnarchuk, M. S., and Essex, J. W. (2015). Water Sites, Networks, And Free Energies with Grand Canonical Monte Carlo. J. Am. Chem. Soc., 137(47):14930-14943.

[39] Ross, G. A., Bruce Macdonald, H. E., Cave-Ayland, C., Cabedo Martinez, A. I., and Essex, J. W. (2017). Replica-Exchange and Standard State Binding Free Energies with Grand Canonical Monte Carlo. J. Chem. Theory Comput., 13(12):6373-6381.

[40] Sasmal, S., Gill, S. C., Lim, N. M., and Mobley, D. L. (2020). Sampling conformational changes of bound ligands using Nonequilibrium Candidate Monte Carlo. J. Chem. Theory Comput., page acs.jctc.9b01066.

[41] Schlessman, J. L., Abe, C., Gittis, A., Karp, D. A., Dolan, M. A., and García-Moreno E., B. (2008). Crystallographic Study of Hydration of an Internal Cavity in Engineered Proteins with Buried Polar or Ionizable Groups. Biophysical Journal, 94(8):3208-3216. 
[42] Stöckmann, H., Bronowska, A., Syme, N. R., Thompson, G. S., Kalverda, A. P., Warriner, S. L., and Homans, S. W. (2008). Residual Ligand Entropy in the Binding of $p$-Substituted Benzenesulfonamide Ligands to Bovine Carbonic Anhydrase II. J. Am. Chem. Soc., 130(37):12420-12426.

[43] Takano, K., Yamagata, Y., and Yutani, K. (2003). Buried water molecules contribute to the conformational stability of a protein. Protein Engineering, Design and Selection, 16(1):5-9.

[44] Wang, J., Wolf, R. M., Caldwell, J. W., Kollman, P. A., and Case, D. A. (2004). Development and testing of a general amber force field. J. Comput. Chem., 25(9):1157-1174.

[45] Woo, H.-J., Dinner, A. R., and Roux, B. (2004). Grand canonical Monte Carlo simulations of water in protein environments. The Journal of Chemical Physics, 121(13):6392-6400.

[46] Young, T., Abel, R., Kim, B., Berne, B. J., and Friesner, R. A. (2007). Motifs for molecular recognition exploiting hydrophobic enclosure in protein-ligand binding. PNAS, 104(3):808-813.

[47] Yu, B., Blaber, M., Gronenborn, A. M., Clore, G. M., and Caspar, D. L. D. (1999). Disordered water within a hydrophobic protein cavity visualized by x-ray crystallography. Proceedings of the National Academy of Sciences, 96(1):103-108. 
367 8.1 Supplementary tables 
Table S1. Acceptance ratios of the replicate simulations at different NCMC step amounts for the MUP-1 system. Each simulation was run for 10,000 iterations. The number of NCMC steps were varied from 1250 to 30000 steps and the number of MD steps was 1000 steps in all cases

\begin{tabular}{|c|c|c|c|c|c|c|c|c|c|c|c|c|}
\hline$n$ NCMC steps & Replicate 1 & Replicate 2 & Replicate 3 & Replicate 4 & Replicate 5 & Replicate 6 & Replicate 7 & Replicate 8 & Replicate 9 & Replicate 10 & Replicate 11 & Replicate 12 \\
\hline 1250 & 0.001 & 0.002 & 0.001 & 0.001 & 0.002 & 0.001 & 0.001 & 0.001 & 0.001 & 0.002 & & \\
\hline 2500 & 0.004 & 0.004 & 0.002 & 0.002 & 0.005 & 0.003 & 0.003 & 0.004 & 0.004 & 0.003 & 0.003 & \\
\hline 5000 & 0.011 & 0.012 & 0.009 & 0.010 & 0.011 & 0.010 & 0.012 & 0.010 & 0.011 & 0.012 & & \\
\hline 7500 & 0.016 & 0.028 & 0.020 & 0.018 & 0.012 & 0.024 & 0.028 & 0.032 & 0.032 & 0.024 & 0.028 & 0.012 \\
\hline 10000 & 0.036 & 0.028 & 0.008 & 0.036 & 0.020 & 0.020 & 0.016 & 0.036 & 0.024 & 0.028 & 0.028 & \\
\hline 15000 & 0.012 & 0.040 & 0.020 & 0.048 & 0.044 & 0.016 & 0.028 & 0.032 & 0.036 & 0.040 & & \\
\hline 20000 & 0.028 & 0.040 & 0.030 & 0.024 & 0.044 & 0.044 & 0.020 & 0.016 & 0.036 & 0.044 & 0.032 & 0.024 \\
\hline 30000 & 0.036 & 0.036 & 0.032 & 0.020 & 0.032 & 0.036 & 0.016 & 0.016 & 0.028 & 0.024 & 0.036 & 0.024 \\
\hline
\end{tabular}


Table S2. Increasing the number of NCMC steps generally increases the acceptance rate of all moves in the MUP-1 protein-ligand system. Here is the average acceptance rate of all moves and average number of force evaluations across 10-12 replicates for the buried cavity in MUP-1 system to become hydrated. Each simulation was run for 10000 BLUES iterations, where each iteration consisted of a single NCMC+MD move. The number of MD steps in all cases was 1000 MD steps per iteration.

\begin{tabular}{lll}
\hline $\boldsymbol{n}$ NCMC steps & Average acceptance rate of all moves & Average number of force evaluations \\
\hline 1250 & $0.1 \%$ & $7.9 \mathrm{E}+06$ \\
2500 & $0.3 \%$ & $2.6 \mathrm{E}+06$ \\
5000 & $1.1 \%$ & $1.1 \mathrm{E}+06$ \\
7500 & $2.3 \%$ & $9.4 \mathrm{E}+05$ \\
10000 & $2.5 \%$ & $1.2 \mathrm{E}+06$ \\
15000 & $3.2 \%$ & $1.7 \mathrm{E}+06$ \\
20000 & $3.2 \%$ & $2.3 \mathrm{E}+06$ \\
30000 & $2.8 \%$ & $2.5 \mathrm{E}+06$ \\
\hline
\end{tabular}

Table S3. Acceptance ratios of all attempted moves for each replicate simulation of the HSP90 protein-ligand system. Shown is the acceptance ratio for four replicate simulations. The average acceptance rate across all four replicates is 0.002 . It took an average of $1693 \mathrm{BLUES}$ iterations to hydrate the cavity.

\begin{tabular}{lllll}
\hline $\boldsymbol{n}$ NCMC steps & Replicate $\mathbf{1}$ & Replicate $\mathbf{2}$ & Replicate $\mathbf{3}$ & Replicate 4 \\
\hline 2500 & 0.004 & 0.002 & 0.002 & 0.002 \\
\hline
\end{tabular}

